\title{
DIDELPHIC UTERUS WITH TERM PRIMIGRAVIDA
}

Dwarakanath. L, Lakshmikantha.G, Musarrat Yamani, Misbah Anjum

1. Associate Professor, Department of Obstetrics \& Gynaecology, Sri Siddhartha Medical College, Tumkur, Karnataka, India.

2. Assistant Professor, Department of Obstetrics \& Gynaecology, Sri Siddhartha Medical College, Tumkur, Karnataka, India.

3. Resident, Department of Obstetrics \& Gynaecology, Sri Siddhartha Medical College, Tumkur, Karnataka, India.

4. Resident, Department of Obstetrics \& Gynaecology, Sri Siddhartha Medical College, Tumkur, Karnataka, India.

\section{CORRESPONDING AUTHOR}

Dr. Lakshmikantha.

G, \#249, $3^{\text {rd }}$ A main,

Near Amba Bhavani Circle,

NE of NR Mohalla, Mysore,

Karnataka, India 570007

E-mail: drlakshmikanthag@yahoo.co.in

Ph: 00919740810611

INTRODUCTION: Urogenital differentiation in the embryo is a complex process involving genetic, hormonal \& environmental influences. The prevalence of mullerian anomalies is estimated as $0.1 \%$ to $3 \%$. American Fertility Society (AFS) classifies mullerian anomalies in to seven types -class I. segmental or complete agenesis or hypoplasia, class II. Unicornuate, class III.Didelphys, class IV.Bicornuate, class V.Septate, class VI. Arcuate, class VII.DES (Di ethyl stilboestrol) related. Didelphys constitutes approximately $11 \%$ of mullerian anomalies and it results from complete failure of fusion of mullerian ducts (1). Mullerian anomaly is responsible as one of the causes for recurrent pregnancy loss and there may be progressive improvement in further pregnancies. Here is a rare case of primigravida with didelphic uterus who presented at term gestation.

CASE REPORT: A 25 years lady presented as primigravida at term gestation in labour. Her past menstrual history revealed menorrhagia. On examination she was pale with stable vitals, systemic examination revealed no abnormalities. A single live fetus at term gestation with breech presentation was noted. On per vaginal examination two separate vagina \& two cervices with thick meconium stained liquor from left vagina was noted. Left cervix was $2 \mathrm{~cm}$ dilated \& station was at -3 . Routine investigations revealed no abnormality. Emergency lower segment cesarean section was done in view of primigravida at term with breech presentation with mullerian anomaly \& alive term female baby weighing $2.6 \mathrm{~kg}$ was delivered from the left uterus. Uterus exteriorized \& inspected for anomaly. Postoperative period uneventful.

DISCUSSION: Two separate uterine bodies each associated with their own fallopian tube and cervix characterize the Didelphic uterus. The diagnosis is typically made when a speculum examination reveals two cervices. Most if not all patients with uterine didelphys will have a longitudinal vaginal septum (2). Conversely most patients with longitudinal vaginal septum will have a didelphic uterus. The didelphic uterus is accepted as having highest rate of successful 
pregnancy of all the uterine anomalies (next only to arcuate uterus). Heinonen PK stated that didelphic uterus has relatively better obstetric outcome, rarely needs metroplasty (3). One study reported a fetal survival rate of $64 \%$ without metroplasty and another $57 \%$ rate of successful pregnancy (4). In our case the pregnancy had reached term gestation except for malpresentation which was the reason why she underwent cesarean section lest she could had been allowed for vaginal delivery. Most current authors believe that most patients with didelphic uterus have adequate reproductive outcomes. Most women with a didelphic uterus do not need special treatment for the condition, but generally women with this condition should make sure to work closely with a doctor during pregnancy to watch for signs of preterm labor or other risks to the baby(5). In women who have two fully developed uteri, pregnancy might be completely normal -- and you might have even seen news articles on rare cases of women with a didelphic uterus getting pregnant in both uteri with different due dates. (6). The unification surgery is contraindicated or at least infrequently indicated. The unification metroplasty can be very technically difficult with disappointing results especially when cervical unification is attempted, which may result in cervical incompetence or cervical stenosis. Surgical interventions are not typically warranted except in cases where longitudinal septum leads to dyspareunia.

CONCLUSION: Favourable obstetric outcome is usual without corrective surgery for didelphic uterus, rather corrective surgery is contraindicated as the results of it is disappointing.

\section{REFERENCES:}

1. Nahum GG. Uterine anomalies. How common are they, and what is their distribution among subtypes?. J Reprod Med. Oct 1998;43(10):877-87. [Medline].

2. Heinonen P.K, Sarikoski S, Pystonen P. Reproductive performance of women with uterine anomalies. Acta obstt Gynecol 1982;61:157-162.

3. Heinonen P K (1984) : Uterus didelphys: A report of 26 cases. Europ. J. Obstet. Gynec. Reprod. Biol., 17/55, 345-350.

4. Musich J R, Behrmen S J, Obstetric outcome before and after metroplasty in women with uterine anomalies, Obstet Gynecol, 1978; 52: 63-66.

5. Cooney, Michael J., Carol B. Benson, and Peter M. Doubliet, "Outcome of pregnancies in women with uterine duplication anomalies." Journal of Clinical Ultrasound 6 Dec 1998. Accessed 14 Mar 2008.

6. Heinonen, Pentti K., "Clinical implications of the didelphic uterus: long-term follow-up of 49 cases." European Journal of Obstetrics \& Gynecology and Reproductive Biology Aug 2000. 183-190. Accessed 8 Dec 2007. 


\section{CASE REPORT}

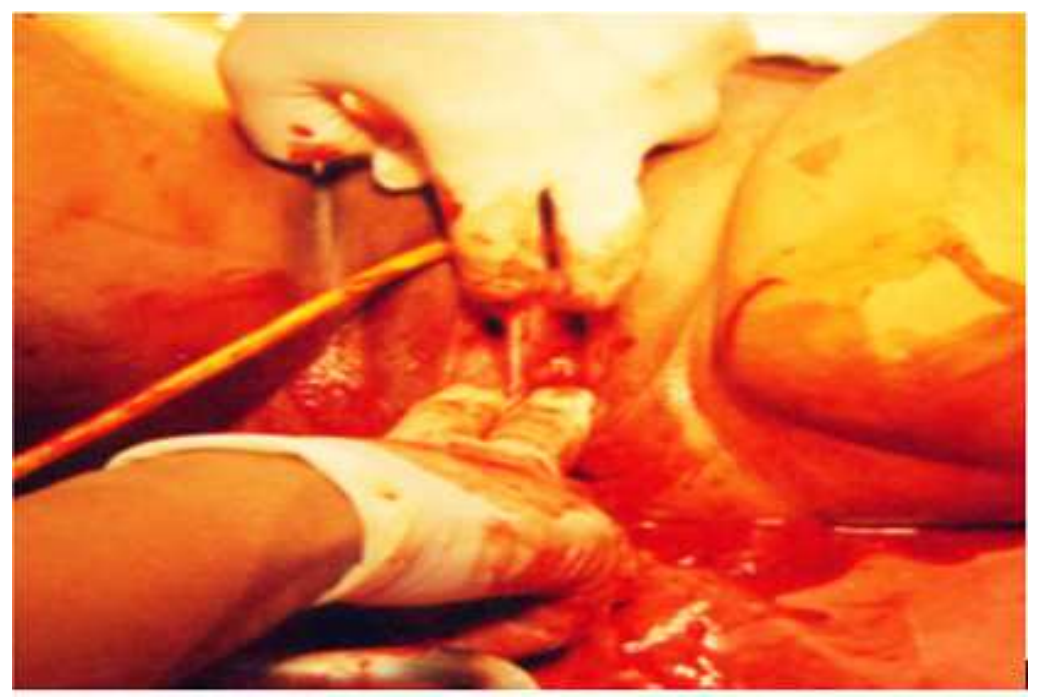

Fig. 1 - Vaginal examination showing two separate vagina.

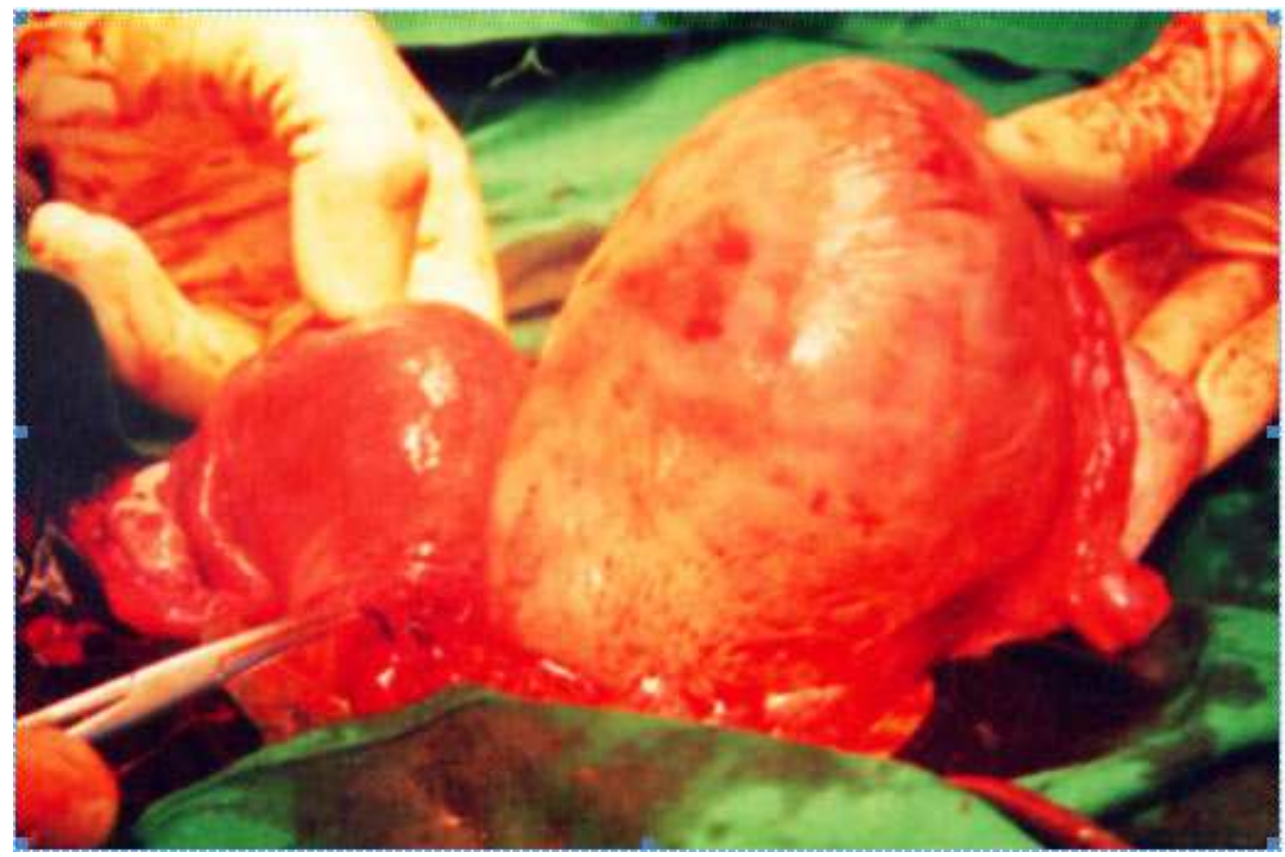

Fig. 2 - Didelphic uterus. 